\title{
ACCURATE HARTREE-FOCK-SLATER CALCULATIONS ON SMALL DIATOMIC MOLECULES WITH THE FINITE-ELEMENT METHOD
}

\author{
D. HEINEMANN, B. FRICKE and D. KOLB \\ Physics Department, University of Kassel, D-3500 Kassel, Federal Republic of Germany
}

Received 13 January 1988

\begin{abstract}
We report on the self-consistent field solution of the Hartree-Fock-Slater equations using the finite-element method for the three small diatomic molecules $\mathrm{N}_{2}, \mathrm{BH}$ and $\mathrm{CO}$ as examples. The quality of the results is not only better by two orders of magnitude than the fully numerical finite difference method of Laaksonen et al. but the method also requires a smailer number of grid points.
\end{abstract}

Besides the standard basis set expansion methods [1] for solving the self-consistent Hartree-Fock equations for molecules, the fully numerical finitedifference method has recently been introduced by Laaksonen, Pyykkö and Sundholm. In a series of papers [2-4] they have shown that this method can be used with high accuracy to solve the same physical problem. Their examples were the solution of the Hartree-Fock-Slater equations for small diatomic molecules (a first step towards relativistic calculations has also been taken by the same authors [5]). The main disadvantage of this method is the need for a large number of points to achieve high accuracy.

The finite-element method represents an alternative approach to solve the same differential equations. In two preceding papers we have shown that the finite-element method can be adapted to quantum mechanical problems. The one-electron systems $\mathrm{H}_{2}^{+}$and $\mathrm{HHe}^{2+}[6]$ as well as the simplest two-electron system $\mathrm{H}_{2}$ [7] have been solved with considerable success.

In the finite-element method the space is divided into a number of elements. On each of these elements basis functions are chosen. Hence from a practical point of view, the finite-element method is a basis set method, but no global basis functions are chosen as in standard basis set expansion methods. The local basis functions on each element are polynomials with free expansion coefficients. The size, the shape and the number of elements as well as the order of the polynomials are the "parameters" which can be changed in this method. Once the final choice is made a matrix diagonalisation with respect to the free expansion coefficients leads to the solution of the differential equations.

In the case of the Hartree-Fock-Slater equations

$\frac{1}{2} \nabla^{2} \Psi(\boldsymbol{r})+V(\boldsymbol{r}) \Psi(\boldsymbol{r})=\epsilon \Psi(\boldsymbol{r})$,

with

$$
\begin{aligned}
& V(\boldsymbol{r})=V^{\mathrm{nuc}}(\boldsymbol{r})+V^{\mathrm{c}}(\boldsymbol{r})+V^{\mathrm{x}}(\boldsymbol{r}), \\
& V^{\mathrm{nuc}}(\boldsymbol{r})=-Z_{1} /\left|\boldsymbol{r}_{1}-\boldsymbol{r}\right|-Z_{2} /\left|\boldsymbol{r}_{2}-\boldsymbol{r}\right|, \\
& V^{\mathrm{c}}(\boldsymbol{r})=\int \frac{\rho\left(\boldsymbol{r}^{\prime}\right)}{\left|\boldsymbol{r}-\boldsymbol{r}^{\prime}\right|} \mathrm{d}^{3} r^{\prime}, \\
& V^{\mathrm{x}}(\boldsymbol{r})=-\frac{3}{2} \alpha[(3 / \pi) \rho(\boldsymbol{r})]^{1 / 3},
\end{aligned}
$$

the finite-element method can be applied if these equations are written as the functional

$$
\begin{aligned}
& \frac{1}{2} \int|\nabla \Psi|^{2} \mathrm{~d}^{3} r+\int V(\boldsymbol{r})|\Psi|^{2} \mathrm{~d}^{3} r \\
& -\epsilon \int|\Psi|^{2} \mathrm{~d}^{3} r
\end{aligned}
$$

which is minimised by the solution of the matrix equation

$\boldsymbol{H} \boldsymbol{x}=\epsilon \mathbf{S} \boldsymbol{x}$.

$\mathbf{H}$ is the Hamiltonian matrix, $\mathbf{S}$ the norm matrix and 
$x$ represents the values of the eigenfunctions at the grid points.

The solution of the Poisson equation which connects the electron charge density and the molecular potential is obtained by solving for the functional

$\frac{1}{2} \int\left(\nabla V^{c}\right)^{2} \mathrm{~d}^{3} r-4 \pi \int \rho V^{\mathrm{c}} \mathrm{d}^{3} r$

which leads to the matrix equation

$\mathrm{D} y=d$.
$D, \boldsymbol{d}$ and $\boldsymbol{y}$ are the analogs of $\mathrm{H}, \mathbf{S}$ and $\boldsymbol{x}$ in eq. (3). To actually calculate the matrix elements in eqs. (3) and (5) we choose prolate spheroidal coordinates and divide the space so as to be equidistant in these coordinates. The main problem, of course, is to have a sufficient number of elements in the vicinity of the nuclei where the large charge density of the inner electrons is concentrated. This shows up especially for large internuclear distances where the charge density is a strongly peaked function of the angular variable.

Using this finite-element method we have solved

Table 1

Total energy and energy eigenvalues for the systems $\mathrm{N}_{2}, \mathrm{BH}$ and $\mathrm{CO}$. The Slater exchange parameter was 0.7 for all calculations. For the largest grids the last figure is uncertain. All values are given in au

$\mathrm{N}_{2}$, internuclear distance $R=2.07 \mathrm{au}$

ref. [5] this work, fifth-order polynomials

\begin{tabular}{|c|c|c|c|c|c|}
\hline points & 5989 & 2601 & 3136 & 3721 & 4356 \\
\hline & -108.346622 & -108.346605 & -108.3466076 & -108.3466086 & -108.3466090 \\
\hline$\epsilon\left(1 \sigma_{g}\right)$ & -13.981070 & -13.9810680 & -13.98106828 & -13.98106837 & -13.98106840 \\
\hline$\epsilon\left(1 \sigma_{u}\right)$ & -13.979661 & -13.9796581 & -13.97965838 & -13.97965847 & -13.97965850 \\
\hline$\epsilon\left(2 \sigma_{u}\right)$ & -0.460725 & -0.46072506 & -0.46072505 & -0.46072505 & -0.46072505 \\
\hline$\epsilon\left(1 \pi_{\mathrm{u}}\right)$ & -0.404235 & -0.40423461 & -0.40423462 & -0.40423462 & -0.40423462 \\
\hline \multicolumn{6}{|c|}{$\begin{array}{l}\mathrm{N}_{2} \text {, internuclear distance } R=2.07 \text { au } \\
\text { this work, sixth-order polynomials }\end{array}$} \\
\hline points & 2401 & 3025 & 3721 & 4498 & \\
\hline & -108.3466090 & -108.34660925 & -108.34660932 & -108.34660934 & \\
\hline$\epsilon\left(1 \sigma_{g}\right)$ & -13.9810686 & -13.98106844 & -13.98106844 & -13.98106844 & \\
\hline \multirow[t]{3}{*}{$\epsilon\left(3 \sigma_{g}\right)$} & -0.35005852 & -0.35005852 & -0.35005853 & -0.35005852 & \\
\hline & \multicolumn{2}{|l|}{ ref. [ 5 ] } & \multicolumn{2}{|c|}{ this work, fifth-order polynomials } & \\
\hline & $\mathrm{BH}$ & $\mathrm{CO}$ & $\mathrm{BH}$ & $\mathrm{CO}$ & \\
\hline points & 15609 & 5989 & 4356 & 4356 & \\
\hline$R$ & 2.366 & 2.13 & 2.366 & 2.13 & \\
\hline$E_{\mathrm{T}}$ & -24.808852 & -112.129925 & -24.8088515 & -112.129915 & \\
\hline$\epsilon(1 \sigma)$ & -6.5323604 & -18.744146 & -6.53236004 & -18.7441431 & \\
\hline$\epsilon(2 \sigma)$ & -0.4078652 & -9.911347 & -0.40786519 & -9.9113461 & \\
\hline
\end{tabular}


the three standard systems $\mathrm{N}_{2}, \mathrm{BH}$ and $\mathrm{CO}$ in order to ascertain whether or not this approach is a useful alternative to the finite-difference method.

Table 1 presents the energy eigenvalues as well as the total energies of $\mathrm{N}_{2}$ for different grid sizes and fifth- and sixth-order two-dimensional polynomials as basis functions on the elements.

For 2401 points the accuracy of the sixth-order polynomial grid is comparable to that of the 4356-point fifth-order polynomial grid. This shows clearly the advantage of high-order polynomials as basis functions for the finite-element method in order to achieve results of high precision.

To compare the current results for $\mathrm{N}_{2}$ with those of Laaksonen et al. [4,5] we emphasise that their number of points is the number of points in the half box, whereas our number of points refers to the whole box. Laaksonen et al. give an absolute error of $1 \times 10^{-5}$ au for the total energy. This same accuracy is reached in our calculations with 2601 points and fifth-order polynomials. The error in the total energy attained with the 2401-point sixth-order grid is about $5 \times 10^{-7}$ au. The results achieved with the 4489-point sixth-order grid are probably correct to $10^{-8}$ au. This value is two to three significant figures better than the values of Laaksonen et al., who used about a factor of two more points. Values for the systems BH and $\mathrm{CO}$ are given at the end of table 1 .

This comparison shows that the finite-element method is capable of solving the Hartree-FockSlater equations to an accuracy never reached before and thus is more than an alternative to all other methods. An attempt to achieve very accurate values for the Hartree-Fock equations as a bench mark for the quality of quantum chemical calculations is our next goal.

This work was partially supported by the Deutsche Forschungsgemeinschaft (DFG).

\section{References}

[1] H.F. Schaefer III, Quantum chemistry (Clarendon Press, Oxford, 1984).

[2] L. Laaksonen, P. Pyykkö and D. Sundholm, Intern. J. Quantum Chem. 23 (1983) 319.

[3] L. Laaksonen, D. Sundholm and P. Pyykkö, Chem. Phys. Letters 96 (1983) 1.

[4] L. Laaksonen, D. Sundholm and P. Pyykkö, Intern. J. Quantum Chem. 27 (1985) 601.

[5] L. Laaksonen, D. Sundholm and P. Pyykkö, Computer Phys. Rept. 4 (1986) 313.

[6] W. Schulze and D. Kolb, Chem. Phys. Letters 122 (1985) 271.

[7] D. Heinemann, D. Kolb and B. Fricke, Chem. Phys. Letters 137 (1987) 180. 